Quim. Nova, Vol. 34, No. 2, 284-288, 2011

\title{
GLICOALCALOIDES ANTIFÚNGICOS, FLAVONOIDES E OUTROS CONSTITUINTES QUÍMICOS DE Solanum asperum
}

\author{
Francisco das Chagas L. Pinto, Daniel Esdras de A. Uchoa, Edilberto R. Silveira, Otília Deusdênia L. Pessoa* e Raimundo \\ Braz-Filho\# \\ Departamento de Química Orgânica e Inorgânica, Centro de Ciências, Universidade Federal do Ceará, CP 12200, 60021-940 \\ Fortaleza - CE, Brasil
}

Fernanda M. e Silva, Phellipe N. E. T. Theodoro e Laila S. Espíndola

Faculdade de Ciências da Saúde, Universidade de Brasília, CP 4478, 70919-970 Brasília - DF, Brasil

Recebido em 7/6/10; aceito em 11/8/10; publicado na web em 26/11/10

\begin{abstract}
ANTIFUNGAL GLYCOALKALOIDS, FLAVONOIDS AND OTHER CHEMICAL CONSTITUENTS OF Solanum asperum Rich (Solanaceae). Two glycoalkaloids: solamargine and solasonine; three flavonoids: tiliroside, $7-O$ - $\alpha$-L-ramnopyranosyl-kaempferol and 3-O-[B-D-glucopyranosyl-(1 $\rightarrow 6)-\alpha$-L-ramnopyranosyl]-7- $O$ - $\alpha$-L-ramnopyranosyl-kaempferol, in addition to the tripeptide Leu-Ile-Val, the aminoacid proline and the eicosanoic acid were isolated from Solanum asperum (Solanaceae). The structures of all compounds were determined by interpretation of their spectra (IR, MS, ${ }^{1} \mathrm{H}$ and ${ }^{13} \mathrm{C}$ NMR) and comparison with the literature data. All compounds, except the glycoalkaloids, are being reported for the first time for $S$. asperum. Solasonine showed strong activity (MIC $<0.24 \mu \mathrm{g} / \mathrm{mL}$ ) against four filamentous fungi species of the genera Microsporum and Trichophyton.
\end{abstract}

Keywords: Solanum asperum; antifungal glycoalkaloids; flavonoids.

\section{INTRODUÇÃO}

A família Solanaceae é representada por aproximadamente 2000 espécies distribuídas em 95 gêneros, sendo o taxon Solanum o mais representativo, com aproximadamente 1500 espécies e 5000 epítetos. As plantas que representam este gênero estão distribuídas preferencialmente nas áreas tropicais e subtropicais do planeta, sendo a América do Sul, o centro de maior diversidade e distribuição. ${ }^{1}$ Uma grande fração do total de espécies descritas pode ser encontrada no Brasil, particularmente, na região Nordeste, onde são catalogadas cerca de 350 espécies. ${ }^{2}$ Várias plantas deste gênero são popularmente conhecidas como "jurubebas", muitas das quais são largamente utilizadas na medicina popular, especialmente no tratamento de doenças da pele ou doenças relacionadas ao fígado e baço. ${ }^{3,4}$

As plantas do gênero Solanum destacam-se pela capacidade de biossintetizar esteroides e alcaloides, livres ou na forma de heterosídeos, metabólitos secundários estruturalmente diversificados e complexos. ${ }^{5,6}$ Estes compostos, em geral, são de interesse terapêutico, visto que apresentam um grande leque de atividades, como citotóxica, ${ }^{5}$ anticâncer, ${ }^{7}$ anti-inflamatória, ${ }^{8}$ antiulcerogênica ${ }^{9}$ e moluscicida. ${ }^{10}$ Além disso, são também responsáveis pela resistência natural das espécies em seu ecossistema. ${ }^{11}$ Compostos fenólicos, principalmente flavonas e flavonóis, incluindo seus heterosídeos, são também identificados com frequência em espécies de Solanum. ${ }^{1,12}$

Como parte de um estudo multidisciplinar, cujo objetivo é investigar plantas da família Solanaceae, na busca por compostos bioativos, especialmente com atividade anticâncer, já foram isoladas várias lactonas esteroidais. ${ }^{13,14}$ Neste trabalho é relatado o resultado obtido com a prospecção química de Solanum asperum Rich, popularmente conhecido como "coça-coça" ou "russara". As plantas desta espécie apresentam porte arbustivo e todas as suas partes são pulverulentas, isto é, ricas em pelos urticantes que se desprendem facilmente e que,

*e-mail: opessoa@ufc.br

"Prof. Honoris causa - UFC, Pesquisador Visitante Emérito em contato com a pele, provocam intensa coceira, daí o nome popular. Concomitante ao estudo químico foi ainda investigado o potencial antifúngico de algumas das substâncias isoladas.

\section{PARTE EXPERIMENTAL}

\section{Procedimentos experimentais gerais}

Os pontos de fusão foram obtidos em equipamento de microdeterminação digital da Mettler Toledo com placa aquecedora FP82HT e central de processamento FP90. As determinações foram realizadas a uma velocidade de aquecimento de $2{ }^{\circ} \mathrm{C} / \mathrm{min}$ e não foram corrigidas. Os espectros no infravermelho foram registrados em espectrômetro Perkin - Elmer Spectrum 1000 FTIR, utilizando pastilhas de KBr. Os espectros de $\mathrm{RMN}{ }^{1} \mathrm{H}$ e ${ }^{13} \mathrm{C}$, uni e bidimensionais, foram obtidos em espectrômetro Bruker Avance DRX-500 (500 MHz para ${ }^{1} \mathrm{H}$ e 125 $\mathrm{MHz}$ para ${ }^{13} \mathrm{C}$ ). Os espectros de massas de baixa resolução foram obtidos em espectrômetro de massa Shimadzu, QP 5000/DI-50, por impacto eletrônico a 70 eV. Nas cromatografias de adsorção utilizouse gel de sílica 60 da Vetec ( 230-400 mesh), enquanto nos fracionamentos cromatográficos por exclusão molecular foi empregado Sephadex LH-20 (Pharmacia). As cromatografias em camada delgada analítica (CCDA) foram realizadas em cromatoplaca de sílica gel (Merck) com indicador de fluorescência $\left(\mathrm{F}_{254}\right)$. As substâncias foram reveladas pela exposição a vapores de iodo, ou por aspersão de solução de vanilina/ácido perclórico/EtOH, seguida de aquecimento em estufa $\left(\approx 100{ }^{\circ} \mathrm{C}\right)$, ou ainda por imersão em solução do reagente Dragendorff.

\section{Material vegetal}

As folhas e frutos de $S$. asperum foram coletados no município de Guaramiranga, Ceará, em agosto de 2007. A autenticação do material vegetal foi realizada pelo Prof. E. P. Nunes do Departamento de Biologia - UFC. Uma exsicata representando a coleta da planta 
encontra-se depositada no Herbário Prisco Bezerra (EAC) da Universidade Federal do Ceará, sob o número de registro 29232.

\section{Extração e isolamento}

Os frutos (74,6 g) e folhas (500 g) de S. asperum, secos e triturados, foram separadamente submetidos à maceração exaustiva com EtOH ( $3 \times 0,5 \mathrm{~L}$ para os frutos e $3 \times 6 \mathrm{~L}$ para as folhas). Após evaporação do solvente, sob pressão reduzida, foram obtidos $6,0 \mathrm{~g}$ (8\%) de extrato dos frutos e 40,0 g (8\%) de extrato das folhas. Os resíduos vegetais de frutos e folhas, foram em seguida, extraídos com uma mistura de $\mathrm{EtOH} / \mathrm{H}_{2} \mathrm{O} / \mathrm{AcOH}$ 90:7:3 (8 L). As soluções resultantes de cada extração foram reduzidas em evaporador rotativo a $1 / 3$ do volume inicial e, em seguida, submetidas à partição liquido/ líquido. A solução dos frutos $(500 \mathrm{~mL})$ foi extraída com $\mathrm{CH}_{2} \mathrm{Cl}_{2}$ ( 3 x $50 \mathrm{~mL}$ ), resultando em 720,0 mg de extrato, enquanto a fase aquosa depois de neutralizada com $\mathrm{NH}_{4} \mathrm{OH}$ forneceu um precipitado (300,0 mg) que, em CCDA, mostrou teste positivo com o reagente Dragendorff. A cromatografia por exclusão molecular em Sephadex LH-20 deste precipitado, usando $\mathrm{MeOH}$ como eluente, resultou no isolamento de um sólido branco amorfo, o qual foi identificado como sendo o glicoalcaloide solamargina $\left(\mathbf{1} ; 26,0 \mathrm{mg}\right.$; p.f. $\left.278-279{ }^{\circ} \mathrm{C}\right)$. $\mathrm{O}$ extrato etanólico dos frutos $(6,0 \mathrm{~g})$ foi dissolvido em $\mathrm{MeOH} / \mathrm{H}_{2} \mathrm{O}$ (7:3) e particionado com hexano, $\mathrm{CH}_{2} \mathrm{Cl}_{2}$ e $\mathrm{AcOEt}(3 \times 100 \mathrm{~mL}$ para cada solvente). As soluções de cada solvente foram reunidas, secas com $\mathrm{Na}_{2} \mathrm{SO}_{4}$ anidro, filtradas e concentradas sob pressão reduzida fornecendo as seguintes frações: hexano (800,0 mg), $\mathrm{CH}_{2} \mathrm{Cl}_{2}(410,0$ mg) e AcOEt (169,6 mg). Estas frações, juntamente com a fração hidrometanólica, foram submetidas ao teste de Dragendorff, porém, somente a fração hidrometanólica $(4,4 \mathrm{~g})$ apresentou teste positivo. Deste modo, este material foi submetido à cromatografia em Sephadex LH-20, utilizando $\mathrm{MeOH}$ como eluente. Ao final, foram obtidas 26 frações de $20 \mathrm{~mL}$ que, após serem monitoradas por CCDA, foram reunidas em 6 frações (A-I a A-VI). A fração A-II (489 mg), após cromatografia flash, usando o sistema de eluição AcOEt/MeOH 6:4, resultou no isolamento de um sólido branco amorfo, identificado como solasonina $\left(\mathbf{2} ; 40,0 \mathrm{mg}\right.$; p.f. $\left.286-287^{\circ} \mathrm{C}\right)$.

O extrato etanólico das folhas $(40,0 \mathrm{~g})$ foi dissolvido em $120 \mathrm{~mL}$ de uma mistura $\mathrm{MeOH} / \mathrm{H}_{2} \mathrm{O}(7: 3)$ e extraído (3 x $100 \mathrm{~mL}$ ) com os solventes hexano, $\mathrm{CH}_{2} \mathrm{Cl}_{2}$ e AcOEt. As frações obtidas foram secas com $\mathrm{Na}_{2} \mathrm{SO}_{4}$ anidro, filtradas e concentradas, obtendo-se as frações hexano (11,26 g), $\mathrm{CH}_{2} \mathrm{Cl}_{2}$ (3,43 g) e AcOEt (1,24 g). A fase hidrometanólica, após ser concentrada, resultou em $(23,04 \mathrm{~g})$ de material. Da fração hexânica $(11,26 \mathrm{~g})$, após sucessivas cromatografias em coluna usando como eluente misturas de hexano/AcOEt, foi isolado o ácido eicosanoico (8,0 mg), por eluição com hexano/AcOEt 7:3. $\mathrm{A}$ fração $\mathrm{CH}_{2} \mathrm{Cl}_{2}(3,34 \mathrm{~g})$ foi submetida à cromatografia em gel de sílica, empregando o sistema de solventes $\mathrm{CH}_{2} \mathrm{Cl}_{2} / \mathrm{MeOH}$, sozinhos ou em gradiente de polaridade crescente. Foram coletadas 40 frações de $10 \mathrm{~mL}$, as quais após monitoramento em CCDA foram reunidas em 8 frações (B-I a B-VIII). A fração B-VII (268,0 mg), obtida por eluição com $\mathrm{CH}_{2} \mathrm{Cl}_{2} / \mathrm{MeOH}$ 1:1, foi submetida à cromatografia de exclusão molecular em Sephadex LH-20 e por eluição com MeOH, fornecendo o heterosídeo tilirosídeo $\left(3 ; 21,0 \mathrm{mg}\right.$; p.f. $\left.264-265^{\circ} \mathrm{C}\right)$. A fração AcOEt (1,24 g), após ser fracionada em Sephadex LH-20, usando $\mathrm{MeOH}$ com eluente, resultou em 22 frações que, depois de monitoradas em CCDA, foram reunidas em 6 frações (C-I a C-VI). A fração C-VI (63,6 mg), após cromatografia flash, empregando como eluente $\mathrm{AcOEt} / \mathrm{MeOH}$ 9:1, forneceu outro heterosídeo, o 7-O- $\alpha$-L-ramnopiranosil-kanferol (4; 15,0 mg; p.f. $206-207{ }^{\circ} \mathrm{C}$ ). A fração AcOEt (1,64 g), proveniente da extração com EtOH/ $\mathrm{H}_{2} \mathrm{O} /$ AcOH 90:7:3 das folhas, após cromatografia de exclusão molecular, utilizando $\mathrm{MeOH}$ como fase móvel, resultou no isolamento de um outro heterosídeo flavonoídico, o 3-O-[B-D-glicopiranosil-( $1 \rightarrow 6)-\alpha$ L-ramnopiranosil]-7- $O$ - $\alpha$-L-ramnopiranosil-kanferol (5, $40 \mathrm{mg}$; p.f. 197-198 $\left.{ }^{\circ} \mathrm{C}\right)$. A fração hidrometanólica $(23,0 \mathrm{~g})$, remanescente da partição do extrato EtOH das folhas, foi submetida à cromatografia gravitacional utilizando como fase móvel os sistemas de solventes $\mathrm{CH}_{2} \mathrm{Cl}_{2} / \mathrm{MeOH} / \mathrm{H}_{2} \mathrm{O}$ 8:2:0,1/ 7:3:0,1/ 6:4:0,1 e $\mathrm{Me}_{2} \mathrm{CO} / \mathrm{MeOH}$ 1:1 (200 mL para cada sistema de solventes). Da fração $\mathrm{CH}_{2} \mathrm{Cl}_{2} / \mathrm{MeOH} /$ $\mathrm{H}_{2} \mathrm{O}$ 7:3:0,1 (1,8g), após sucessivos fracionamentos cromatográficos, empregando Sephadex LH-20 e MeOH como eluente, foi isolado um sólido branco amorfo, caracterizado como sendo um tripeptídeo (6, 9,3 mg, p.f. $181-182{ }^{\circ} \mathrm{C}$ ). Da fração $\mathrm{CH}_{2} \mathrm{Cl}_{2} / \mathrm{MeOH} / \mathrm{H}_{2} \mathrm{O}$ 6:4:0,1 (3,5 g), após sucessivas cromatografias usando Sephadex LH-20, foi obtido o aminoácido, prolina (7, 50,0 mg; p.f. $\left.133-134{ }^{\circ} \mathrm{C}\right)$.

\section{Ensaio da atividade antifúngica}

\section{Micro-organismos}

Os fungos utilizados na avaliação da atividade foram isolados clínicos de dermatófitos das espécies Trichophyton rubrum, T. mentagrophytes, Microsporum canis e M. gypseum, e duas leveduras American Type Culture Collection (ATCC), Candida albicans ATCC 10231 e C. parapsilosis ATCC 22019. Os fungos foram mantidos em tubos de ensaio com meio Agar batata dextrose a $35^{\circ} \mathrm{C}$ (CLSI Documento M27-A2 e M38-A). ${ }^{15,16}$

\section{Concentração inibitória mínima}

Para determinar a concentração inibitória mínima (CIM) foram utilizados os protocolos M27-A2 ${ }^{15}$ para leveduras e o M38- ${ }^{16}$ para fungos filamentosos do Clinical and Laboratory Standards Institute (CLSI). Utilizando-se a técnica de microdiluição em placas de 96 poços foram realizados os cálculos de CIM, sendo utilizado o meio de cultura RPMI (Roswell Park Memorial Institute) 1640 (Sigma ${ }^{\circledR}$ ) como controle negativo e o itraconazol e fluconazol como controles positivos. As amostras foram dissolvidas em dimetilsulfóxido (DMSO). Os testes foram realizados em triplicata, sendo a concentração de DMSO inferior a $5 \%$. O tempo de incubação foi de $48 \mathrm{~h}$ para leveduras e de $72 \mathrm{~h}$ para dermatófitos, à $35^{\circ} \mathrm{C}$. A menor concentração capaz de inibir totalmente o crescimento do fungo foi considerada o valor de CIM.

\section{RESULTADOS E DISCUSSÃO}

A prospecção química dos frutos de $S$. asperum culminou no isolamento dos glicoalcaloides, solamargina (1) ${ }^{17}$ e solasonina (2), ${ }^{18}$ os quais foram recentemente publicados a partir da espécie em estudo, incluindo sua propriedade moluscicida. ${ }^{10}$ Das folhas de $S$. asperum foram isolados os flavonoides, tilirosídeo ${ }^{19}(3), 7-O-\alpha-\mathrm{L}-$ ramnopiranosil-kanferol (4) e 3-O-[ß-D-glicopiranosil-(1 $\rightarrow 6)-\alpha-\mathrm{L}-$ ramnopiranosil]-7-O- $\alpha$-L-ramnopiranosil-kanferol ${ }^{20,21}$ (5), um tripeptídeo (6) e o aminoácido prolina ${ }^{22}$ (7), Figura 1. Além dos compostos indicados acima, foi isolado o ácido eicosanoico, cuja estrutura foi identificada por CG-EM. As estruturas de 1-6 foram determinadas por $\mathrm{RMN}{ }^{1} \mathrm{H}$ e ${ }^{13} \mathrm{C}$, incluindo experimentos bidimensionais, como COSY, HSQC e HMBC, além de IV e EM.

O composto 6 foi isolado como um sólido branco amorfo, p.f. 181-182 ${ }^{\circ} \mathrm{C}$. A fórmula molecular $\mathrm{C}_{17} \mathrm{H}_{33} \mathrm{~N}_{3} \mathrm{O}_{4}$ foi determinada por combinação dos dados de RMN e espectrometria de massa de baixa resolução, que revelou o pico correspondente ao íon molecular $\mathrm{m} / \mathrm{z}$, 343. O espectro no infravermelho apresentou uma banda larga centrada em $3448 \mathrm{~cm}^{-1}$ compatível com vibrações de deformação axial de ligações N-H e O-H que, quando associadas às absorções em 1610,1548 e $1514 \mathrm{~cm}^{-1}$, podem ser atribuídas tanto a amidas como a grupamentos carboxilatos de aminoácidos. O espectro de RMN ${ }^{1} \mathrm{H}\left(500 \mathrm{MHz}, \mathrm{D}_{2} \mathrm{O}\right)$ apresentou sinais em $\delta 3,72(\mathrm{dd}, J=8,4$ e 5,1 


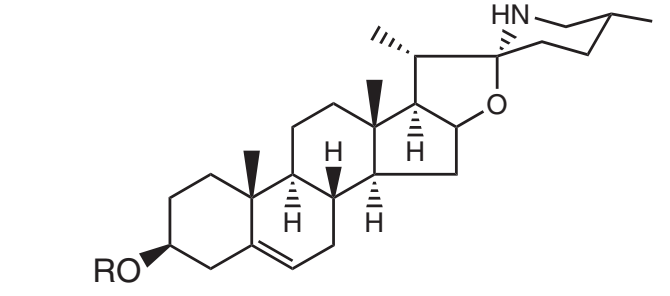

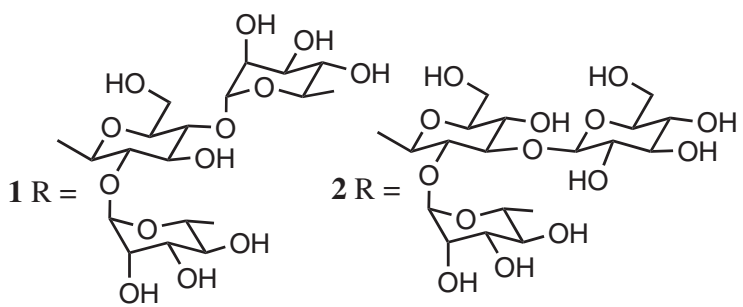<smiles>O=C(/C=C/c1ccc(O)cc1)OCC(O)C(O)OC(O)C(O)COc1c(-c2ccc(O)cc2)oc2cc(O)cc(O)c2c1=O</smiles><smiles>CC1OC(Oc2cc(O)c3c(=O)c(O)c(-c4ccc(O)cc4)oc3c2)C(O)C(O)C1O</smiles>

4

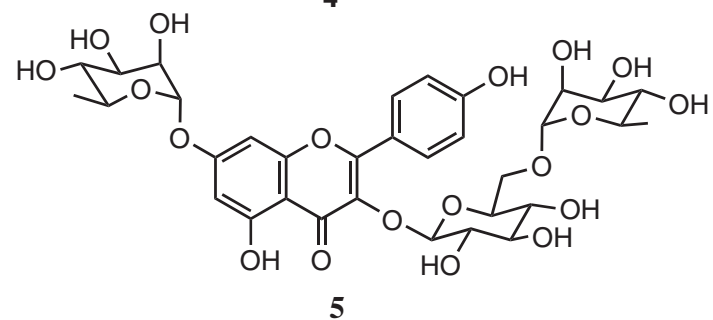<smiles>CCC(C)C(NC(=O)C(N)C(C)C)C(=O)NC(CC(C)C)C(=O)O</smiles>

Figura 1. Estruturas dos constituintes químicos isolados de Solanum asperum

$\mathrm{Hz}, \mathrm{H}-2), 3,66$ (d, $\left.J=3,9 \mathrm{~Hz}, \mathrm{H}-2^{\prime}\right)$ e 3,60 (d, $J=4,3 \mathrm{~Hz}, \mathrm{H}-2^{\prime \prime}$ ) compatíveis com a presença de átomos de hidrogênio ligados a carbonos $\alpha$-carbonílicos nitrogenados, uma série de multipletos em $\delta$ 2,27 (H-3'”); 1,98 (H-3'); 1,73 (H-4); 1,72 (H-3a), 1,69 (H-3b); 1,46 (H-4`a) e 1,25 (H-4`b), referentes a hidrogênios metínicos e metilênicos, além dos sinais em 0,98 (d, $\left.J=7,0 \mathrm{~Hz}, 3 \mathrm{H}-4^{\prime \prime}\right)$; 1,00 (d, $J=7,0 \mathrm{~Hz}, 3 \mathrm{H}-6^{\prime}$ ); 1,03 (d, $\left.J=7,0 \mathrm{~Hz}, 3 \mathrm{H}-5^{\prime \prime}\right) ; 0,96$ (d, $J=5,9$ $\mathrm{Hz}, 3 \mathrm{H}-6) ; 0,95$ (d, $J=5,8 \mathrm{~Hz}, 3 \mathrm{H}-5$ ) e 0,93 (t, $J=7,4 \mathrm{~Hz}, 3 \mathrm{H}-5^{\prime}$ ) correspondentes a seis grupos metilas. No espectro COSY, foram observados correlações que revelaram claramente três sistemas de acoplamentos separados. O espectro de $\mathrm{RMN}{ }^{13} \mathrm{C}\left(125 \mathrm{MHz}, \mathrm{D}_{2} \mathrm{O}\right)$ apresentou sinais correspondentes a dezessete átomos de carbono, incluindo três grupos carboxilas em $\delta 174,3$ e 174,2 (2 x C=O), seis carbonos metínicos, dos quais três $(\delta 60,4 ; 59,6$ e 53,5) são nitrogenados, além de dois grupos metilênicos e seis metilas (Tabela 1). A atribuição inequívoca dos sinais correspondentes aos grupos metilas foi realizada através do experimento TOCSY 1D seletivo, no qual a excitação na frequência do sinal em $\delta 3,72(\mathrm{H}-2)$ exibiu correlação com os hidrogênios metilênicos $\delta 1,72(\mathrm{H}-3 \mathrm{a}) / 1,69(\mathrm{H}-3 \mathrm{~b})$ e metínico $\delta 1,73(\mathrm{H}-4)$, além dos dois metilas em $\delta 0,96$ (3H-6) e 0,95 (3H-5); a irradiação do sinal em $\delta 3,66$ (H-2') mostrou correlação com os hidrogênios metínicos $\delta$ 1,98 (H-3') e metílico $\delta$ 1,00 (3H-6'). De maneira semelhante, a excitação do sinal em $\delta 3,60$ (H-2") revelou a correlação com os sinais dos hidrogênios metílicos em $\delta 1,03$ (3H-4") e 0,98 (3H-5"). A sequência das três unidades de aminoácidos foi confirmada por espectrometria de massa, através dos fragmentos $\mathrm{m} / \mathrm{z}$. 326 (6a), 272 (6b), 242 (6c), 213 (6d), 129 (6e) e 100 (6f) Daltons, cujas estruturas encontram-se esboçadas na Figura 2. Com estes dados, incluindo-se aqueles obtidos dos espectros HSQC e HMBC (Tabela 1), chegou-se à estrutura do tripeptídeo L-Valil-L-IsoleucilL-leucina (Leu-Ile-Val), o qual foi previamente relatado de fonte animal (músculo de salmão). ${ }^{23}$

Tabela 1. Deslocamentos químicos de $\mathrm{RMN}{ }^{1} \mathrm{H}$ e ${ }^{13} \mathrm{C}\left(\mathrm{D}_{2} \mathrm{O}\right)^{*}$ do composto 6

\begin{tabular}{|c|c|c|c|c|}
\hline $\mathbf{C}$ & $\delta_{\mathrm{C}}$ & $\delta_{\mathrm{H}}$ & ${ }^{2} \mathrm{~J}_{\mathrm{CH}}$ & ${ }^{3} \mathrm{~J}_{\mathrm{CH}}$ \\
\hline $\mathrm{C}-1$ & 174,32 & - & $\mathrm{H}-2$ & \\
\hline $\mathrm{CH}-2$ & 53,50 & $3,72(\mathrm{dd}, 8,4,5,1)$ & $2 \mathrm{H}-3$ & $\mathrm{H}-4$ \\
\hline $\mathrm{CH}_{2}-3$ & 39,86 & $1,72(\mathrm{~m}), 1,69(\mathrm{~m})$ & H-2; H-4 & $3 \mathrm{H}-5 ; 3 \mathrm{H}-6$ \\
\hline $\mathrm{CH}-4$ & 24,23 & $1,73(\mathrm{~m})$ & $\begin{array}{c}2 \mathrm{H}-3 ; 3 \mathrm{H}-5 ; \\
3 \mathrm{H}-6\end{array}$ & $\mathrm{H}-2$ \\
\hline $\mathrm{CH}_{3}-5$ & 20,95 & $0,95(\mathrm{~d}, 5,8)$ & $\mathrm{H}-4$ & $2 \mathrm{H}-3$ \\
\hline $\mathrm{CH}_{3}-6$ & 22,09 & $0,96(\mathrm{~d}, 5,9)$ & $\mathrm{H}-4$ & $2 \mathrm{H}-3$ \\
\hline C-1' & 174,23 & - & $\mathrm{H}-2$ & \\
\hline $\mathrm{CH}-2^{\prime}$ & 59,62 & $3,66(\mathrm{~d}, 3,9)$ & & $2 \mathrm{H}-4^{\prime} ; 3 \mathrm{H}-6^{\prime}$ \\
\hline CH-3' & 35,14 & $1,98(\mathrm{~m})$ & H-2'; 2H-4' & $3 \mathrm{H}-5$; $3 \mathrm{H}-6$ \\
\hline $\mathrm{CH}_{2}-4^{\prime}$ & 24,52 & $1,46(\mathrm{~m}), 1,25(\mathrm{~m})$ & & H-2'; 3H-6' \\
\hline $\mathrm{CH}_{3}-5^{\prime}$ & 11,16 & $0,93(t, 7,4)$ & $2 \mathrm{H}-4^{\prime}$ & \\
\hline $\mathrm{CH}_{3}-6^{\prime}$ & 14,74 & $1,00(\mathrm{~d}, 7,0)$ & & H-2'; 2H-4' \\
\hline $\mathrm{C}-1^{\prime \prime}$ & 174,23 & - & $\mathrm{H}-2 "$ & H-3" \\
\hline $\mathrm{CH}-2^{\prime \prime}$ & 60,45 & $3,60(\mathrm{~d}, 4,3)$ & $\mathrm{H}-3 "$ & $3 \mathrm{H}-4 " ; 3 \mathrm{H}-5 " 7$ \\
\hline $\mathrm{CH}-3^{\prime \prime}$ & 29,14 & $2,27(\mathrm{~m})$ & $\begin{array}{c}\text { H-2"; 3H-4"; } \\
3 \mathrm{H}-5 "\end{array}$ & \\
\hline $\mathrm{CH}_{3}-4^{\prime \prime}$ & 16,70 & $0,98(\mathrm{~d}, 7,0)$ & & H-2”; 3H-5” \\
\hline $\mathrm{CH}_{3}-5^{\prime \prime}$ & 18,01 & $1,03(\mathrm{~d}, 7,0)$ & & $\mathrm{H}-2 " ; 3 \mathrm{H}-4 "$ \\
\hline
\end{tabular}

*Deslocamentos químicos $(\delta)$ em ppm. Padrão de hidrogenação determinado através da técnica DEPT $135^{\circ}$. Multiplicidade e constante de acoplamento $(J)$ entre parêntesis. $J$ em Hertz.

Neste trabalho, os compostos $\mathbf{1}$ e $\mathbf{2}$ foram avaliados sobre as leveduras Candida albicans e C. parapsilosis e em fungos filamentosos Microsporum canis, M. gypseum, Trichophyton rubrum e $T$. mentagrophytes (Tabela 2). O glicoalcaloide $\mathbf{1}$ apresentou valores de CIM de $125 \mu \mathrm{g} / \mathrm{mL}$ para leveduras e $62,5 \mu \mathrm{g} / \mathrm{mL}$ para os filamentosos, enquanto o glicoalcaloide 2 foi mais ativo, com valores de 62,5 $\mu \mathrm{g} / \mathrm{mL}$ sobre as leveduras e $0,24 \mu \mathrm{g} / \mathrm{mL}$, sobre os filamentosos. Esses compostos possuem estruturas semelhantes, entretanto o espectro de atividade é diferente. A potente atividade do glicoalcaloide $\mathbf{2}$ para dermatófitos é bastante promissora, sobretudo considerandose que o controle positivo itraconazol apresentou valores de CIM entre 0,5-8,0 $\mu \mathrm{g} / \mathrm{mL}$. Esses resultados são de importância clínica, 
Tabela 2. Valores da concentração inibitória mínima (CIM), em $\mu \mathrm{g} / \mathrm{mL}$ de solamargina (1) e solasonina (2) sobre leveduras e fungos filamentosos

\begin{tabular}{|c|c|c|c|c|c|c|}
\hline Composto & C. albicans & C. parapsilosis & T. rubrum & T. mentagrophytes & M. canis & M. gypseum \\
\hline Solamargina & 125 & 125 & 62,5 & 62,5 & 62,5 & 62,5 \\
\hline Solasonina & 62,5 & 62,5 & 0,24 & 0,24 & 0,24 & 0,24 \\
\hline a'Itraconazol & 0,5 & 0,25 & 0,5 & 1 & 8 & 0,5 \\
\hline Fluconazol & 1 & 1 & 64 & $>64$ & $>64$ & 16 \\
\hline bPMI 100\% & - & - & - & - & - & - \\
\hline
\end{tabular}

${ }^{\mathrm{a}}$ Controles positivos, ${ }^{\mathrm{b}}$ Controle negativo, ${ }^{-}$não ativo.<smiles>CCC(C)C(NC(=O)C(N)C(C)C)C(=O)NC(C#[O+])CC(C)C</smiles>

6a $m / z, 326$ $\left([\mathrm{M}]^{\cdot+}-\mathrm{OH}\right)$<smiles>CCC(C)C(=N)C(=O)NC(CC(C)C)C(=O)O</smiles>

$\left([\mathrm{M}]^{\bullet+}-\mathrm{Me}_{2} \mathrm{CHCH}_{2} \mathrm{CHNH}_{2} \mathrm{COOH}\right)$

6c $m / z, 242$

$\left([\mathrm{M}]^{*+}-\mathrm{Me}_{2} \mathrm{CHCHNH}_{2} \mathrm{CHO}\right)$ e/ou $\left(\mathbf{6 b}-\mathrm{CH}_{2} \mathrm{O}\right)$<smiles>CC(C)CC(=N)C(=O)O</smiles>

6e $m / z, 129$<smiles>CCC(C)C(C=[O+])NC(=O)C(N)C(C)C</smiles>

6d $\mathrm{m} / \mathrm{z} 213$<smiles>CCC(C)C(NC(=O)C(=O)NC(CC(C)C)C(=O)O)C(=O)O</smiles>

6b $m / z 272$ $\left([\mathrm{M}]^{\cdot+}-\mathrm{Me}_{2} \mathrm{C}=\mathrm{CHNH}_{2}\right)$<smiles>CCC(C)C(=N)C(=O)NC(CC(C)C)C(=O)O</smiles>

$m / z 314\left([\mathrm{M}]^{\bullet+}\right)$

Figura 2. Estruturas pontuais de fragmentos gerados no espectrômetro de massa, por impacto eletrônico (EIMS), e utilizados na proposição da estrutura do tripeptídeo Leu-Ile-Val (6)

uma vez que, cerca de $22 \%$ dos pacientes HIV positivos apresentam micoses promovidas principalmente por dermatófitos ${ }^{24,25}$ e $40 \%$ das manifestações cutâneas em transplantados renais são promovidas por fungos. ${ }^{26}$ Este é o primeiro relato da atividade dos glicoalcaloides $\mathbf{1}$ e $\mathbf{2}$ em fungos patógenos humanos, pois são descritos apenas pela ação em fungos endofíticos. ${ }^{27}$ Existe uma predominância de $T$. rubrum como principal agente etiológico em várias regiões geográficas do mundo, como Japão, Estados Unidos, México e Brasil. ${ }^{28}$ No entanto, alguns estudos mostram o aparecimento de outros dermatófitos como principais agentes causadores de infecção, entre eles o T. mentagrophytes e o M. canis..$^{29,30}$ A efetividade do tratamento tópico de infecções fúngicas continua sendo um importante desafio em dermatologia. Apesar do amplo espectro de ação dos antifúngicos tópicos, em dermatófitos e leveduras, altas taxas de recidiva e recorrência de sintomas continuam sendo problemas clínicos importantes. ${ }^{31}$ Infecções nas unhas, difíceis de erradicar, podem apresentar níveis de recorrência de 25 a $40 \%$ dos casos, mesmo em uso de antifúngicos sistêmicos..$^{32}$ Compostos como o glicoalcaloide 2 poderiam ser uma alternativa de opção para o desenvolvimento de formulação tópica para o tratamento de micoses.

\section{CONCLUSÃO}

A prospecção química das folhas e frutos de $S$. asperum resultou no isolamento de uma série de metabólitos secundários pertencentes a classes estruturais distintas como glicoalcaloides, flavonoides e peptídeos. Com exceção dos glicoalcaloides, os demais compostos estão sendo relatados pela primeira vez a partir da espécie, constituindo-se interessante contribuição para o conhecimento químico do gênero. Este é o primeiro relato da atividade dos glicoalcaloides solamargina e solasonina em fungos patógenos humanos. Esse estudo demonstra a importância da investigação antifúngica de produtos naturais de origem vegetal, aliada à preservação da biodiversidade. Soma-se a isto que alternativas quimioterápicas para o tratamento de infecções fúngicas são de fundamental relevância para a saúde pública.

\section{MATERIAL SUPLEMENTAR}

As Figuras 1S a 6S estão disponíveis em http://quimicanova.sbq. org.br, na forma de arquivo PDF, com aceso livre.

\section{AGRADECIMENTOS}

Ao apoio financeiro das instituições de apoio à pesquisa $\mathrm{CNPq}$, CAPES, PRONEX, FUNCAP e FAPDF.

\section{REFERÊNCIAS}

1. Sarmento Silva, T. M.; Carvalho, M. G.; Braz-Filho, R.; Agra, M. F.; Quim. Nova 2003, 26, 517.

2. Agra, M. F.; Bhattacharyya, J. Em Solanaceae IV. Advances in Biology and Utilization; Nee, M.; Symon, D. E.; Lester, R. N.; Jessop, J. P., eds.; Royal Botanic Gardens: Kew, 1999.

3. Matos, F. J. A.; Plantas da Medicina Popular do Nordeste, Edições UFC: Fortaleza, 1999. 
4. Lorenzi, H.; Matos, F. J. A.; Plantas Medicinais do Brasil: Nativas e Exóticas Cultivadas, Instituto Plantarum: Nova Odessa, 2002.

5. Ikeda, T.; Tsumagari, H.; Nohara, T.; Biol. Pharm. Bull. 2003, 26, 1198.

6. Distl, M.; Wink, M.; Potato Res. 2009, 52, 79.

7. Friedman, M.; Lee, K-R.; Kim, H-J.; Lee, I-S.; Kozukue, N.; J. Agric. Food Chem. 2005, 53, 6162.

8. Edwin, J. E.; Edwin, S.; Saini, V.; Deb, L.; Gupta, V. B.; Wate, S. P.; Busari, K. P.; Nat. Prod. Res. 2008, 22, 269.

9. Mallika, J.; Shyamala, D. C. S.; J. Ethnopharmacol. 2006, 104, 156.

10. Silva, T. M. S.; Camara, C. A.; Freire, K. R. L.; Silva, T. G.; Agra, M. F.; Bhattacharyya, J.; J. Braz. Chem. Soc. 2008, 19, 1048.

11. Friedman, M.; J. Agric. Food Chem. 2006, 54, 8655.

12. Hale, A. L.; Reddivari, L.; Nzaramba, M. N.; Bamberg, J. B.; Miller Jr, J. C.; Am. J. Pot. Res. 2008, 85, 332.

13. Veras, M. L.; Bezerra, M. Z. B.; Lemos, T. L. G.; Uchoa, D. E. A.; BrazFilho, R.; Chai, H. B.; Cordell, G. A.; Pessoa, O. D. L.; J. Nat. Prod. 2004, 67, 710.

14. Veras, M. L.; Bezerra, M. Z. B.; Braz-Filho, R.; Pessoa, O. D. L.; Montenegro, R. C.; Pessoa, C.; Moraes, M. O.; Costa-Lotufo, L. V.; Planta Med. 2004, 70, 551.

15. CLSI - Clinical and Laboratory Standards Institute; Reference Method for Broth Dilution Antifungal Susceptibility Testing of Yeasts, Approved Standard, $2^{\text {nd }}$ ed., Document M27-A2, CLSI: Pennsylvania, 2002.

16. CLSI - Clinical and Laboratory Standards Institute; Reference Method for Broth Dilution Antifungal Susceptibility Testing of Filamentous Fungi, Approved Standard, Document M38-A, CLSI: Pennsylvania, 2002.

17. Wanyonyi, A. W.; Chhabra, S. C.; Mkoji, G.; Eilert, U.; Njue, W. M.; Phytochemistry 2002, 59, 79.
18. Ridout, C. L.; Price, K. R.; Coxon, D. T.; Fenwick, G. R.; Pharmazie 1989, 44, 732.

19. Lencina, C.; Pires, V. S.; Gosmann, G.; Taketa, A. T. C.; Schenkel, E. P.; Rev. Bras. Farmacog. 2001, 11, 89.

20. Agrawal, P. K.; Carbon-13 NMR of Flavonoids, Elsevier: Amsterdan, 1989.

21. Silva, T. M. S.; Carvalho, M. G.; Braz-Filho, R.; Quim. Nova 2009, 32, 1119.

22. Pretsch, E.; Bühlmann, P.; Affolter, C.; Structure Determination of Organic Compounds. Tables of Spectral Data, Spring-Verlag: Berlin, 2000.

23. Enari, H.; Takahashi, Y.; Kawarasaki, M.; Tada, M.; Tatsuta, K.; Fisheries Sci. 2008, 74, 911.

24. Porro, A. M.; Yoshioka, M. C. N.; Anais Bras. Dermatol. 2000, 75, 665.

25. Souza, L. B.; Pinto, L. P.; Medeiros, A. M. C.; Junior, R. F. A.; Mesquita, O. J. X.; Pesqui. Odontol. Bras. 2000, 14, 79.

26. Vettorato, G.; Carvalho, A. V. E.; Lecompte, S. M.; Trez, E. G.; Garcia, V. D.; Keitel, E.; Anais Bras. Dermatol. 2003, 78, 282.

27. Gipollini, M. L.; Levey, D. J.; Ecology 1997, 78, 799.

28. Welsh, O.; Welsh, E.; Ocampo-Candiani, J.; Gomez, M.; Vera-Cabrera, L.; Mycoses 2006, 49, 119.

29. Dolenc-Voljc, M.; Mycoses 2005, 48, 181.

30. Panasiti, V.; Devirgiliis, V.; Borrni, R. G.; Mancini, M.; Curzio, M.; Rossi, M.; Bottoni, U.; Calvieri, S.; Med. Mycol. 2007, 45, 57.

31. Pfaller, M. A.; Sutton, D. A.; Diagn. Microbiol. Infect. Dis. 2006, 56, 147.

32. Woodfolk, J. A.; Clin. Microbiol. Rev. 2005, 18, 30. 
GLICOALCALOIDES ANTIFÚNGICOS, FLAVONOIDES E OUTROS CONSTITUINTES QUÍMICOS DE Solanum asperum

Francisco das Chagas L. Pinto, Daniel Esdras de A. Uchoa, Edilberto R. Silveira, Otília Deusdênia L. Pessoa* e Raimundo Braz-Filho"

Departamento de Química Orgânica e Inorgânica, Centro de Ciências, Universidade Federal do Ceará, CP 12200, 60021-940 Fortaleza - CE, Brasil

Fernanda M. e Silva, Phellipe N. E. T. Theodoro e Laila S. Espíndola

Faculdade de Ciências da Saúde, Universidade de Brasília, CP 4478, 70919-970 Brasília - DF, Brasil

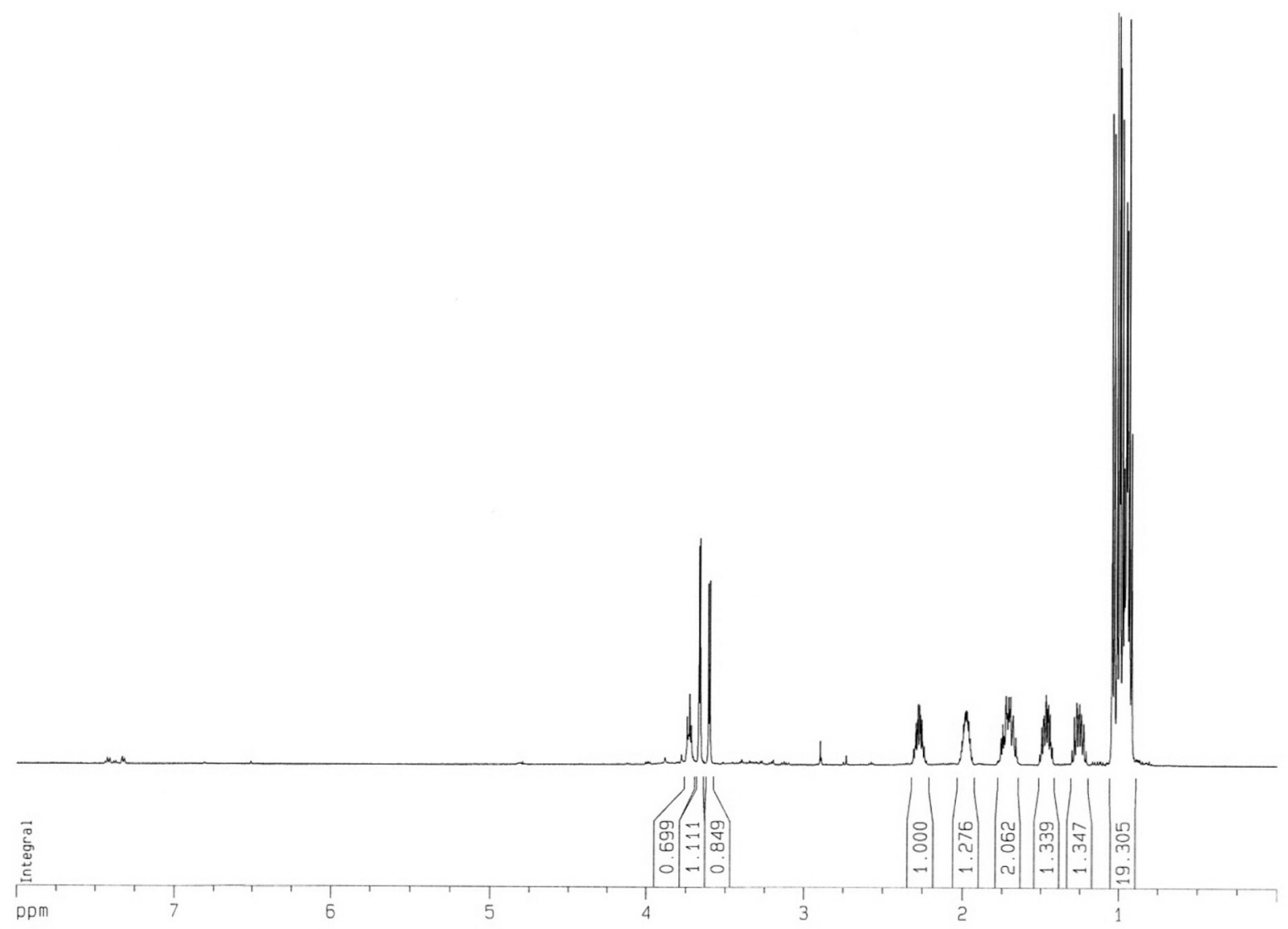

Figura 1S. Espectro de $\mathrm{RMN}^{1} \mathrm{H}\left(500 \mathrm{MHz}, \mathrm{D}_{2} \mathrm{O}\right)$ do composto 6

*e-mail: opessoa@ufc.br

"Prof. Honoris causa - UFC, Pesquisador Visitante Emérito 
言

$$
\text { }
$$

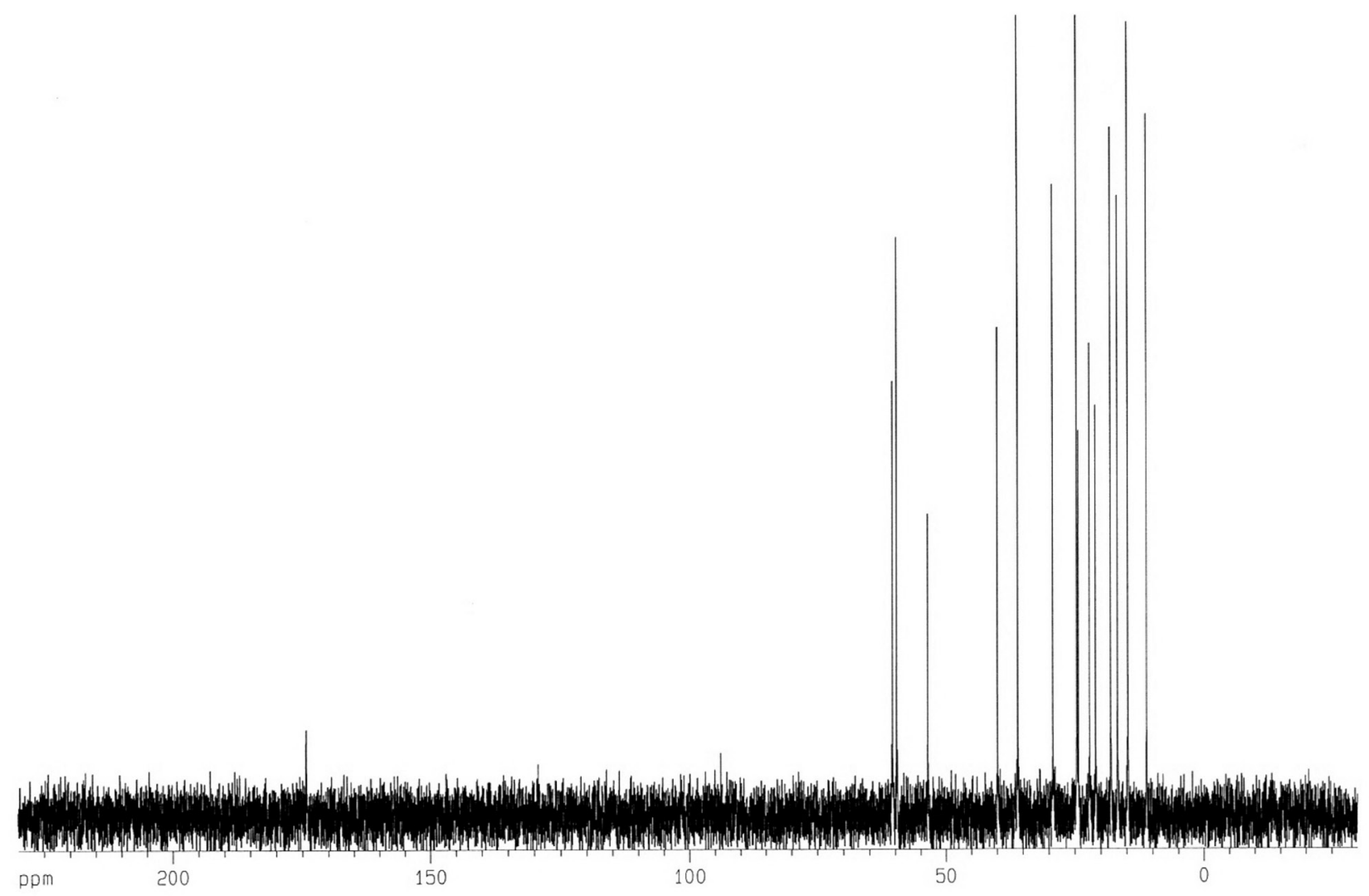

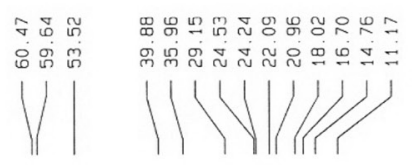

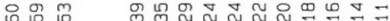

H HWJifle

Figura 2S. Espectro de $\mathrm{RMN}^{13} \mathrm{C}\left(500 \mathrm{MHz}, \mathrm{D}_{2} \mathrm{O}\right)$ do composto 6 


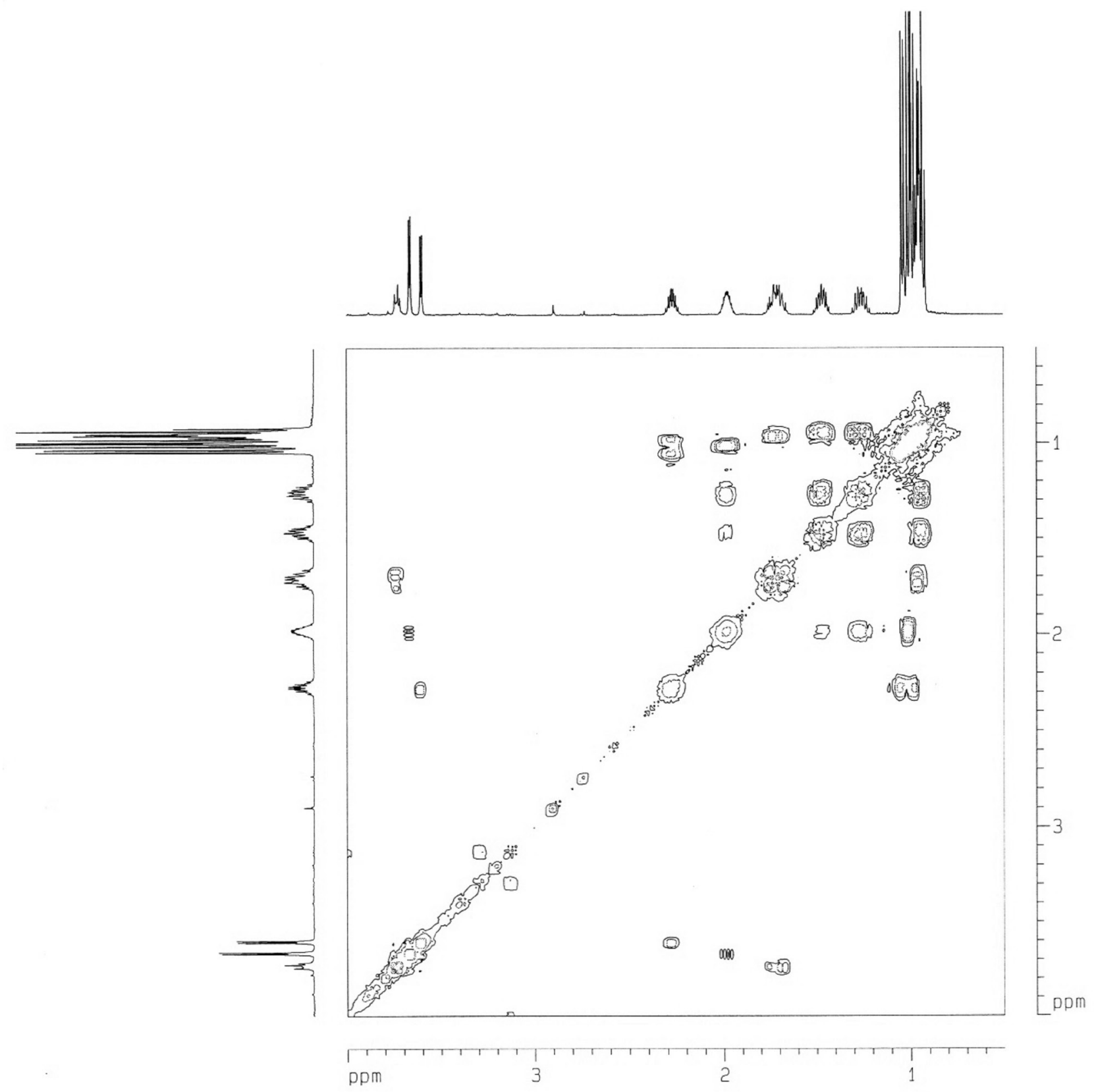

Figura 3S. Espectro de RMN COSY $\left(D_{2} O\right)$ do composto 6 
S4

Pinto et al.

Quim. Nova

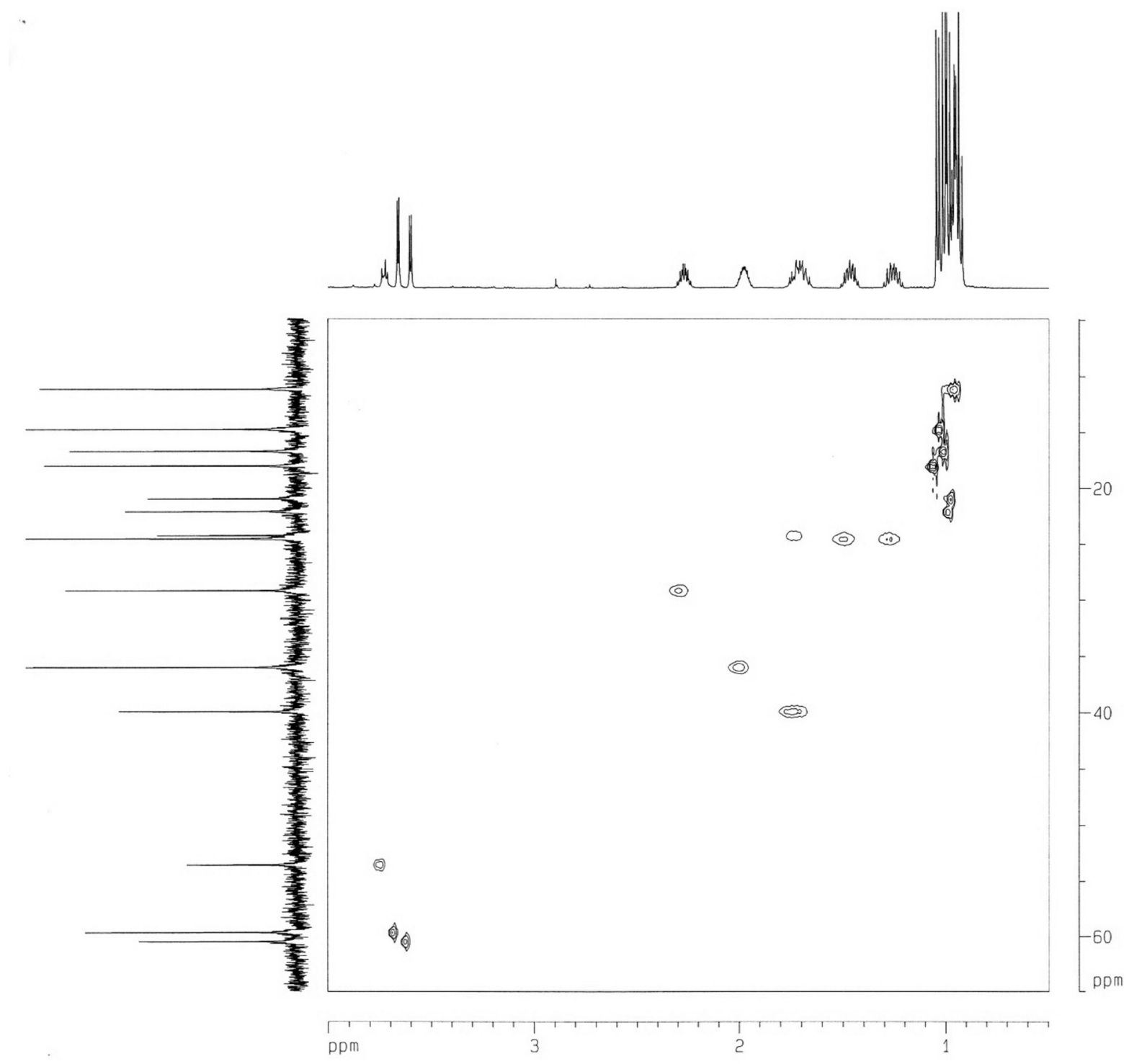

Figura 4S. Mapa de contornos HMQC $\left(D_{2} O\right)$ do composto 6 


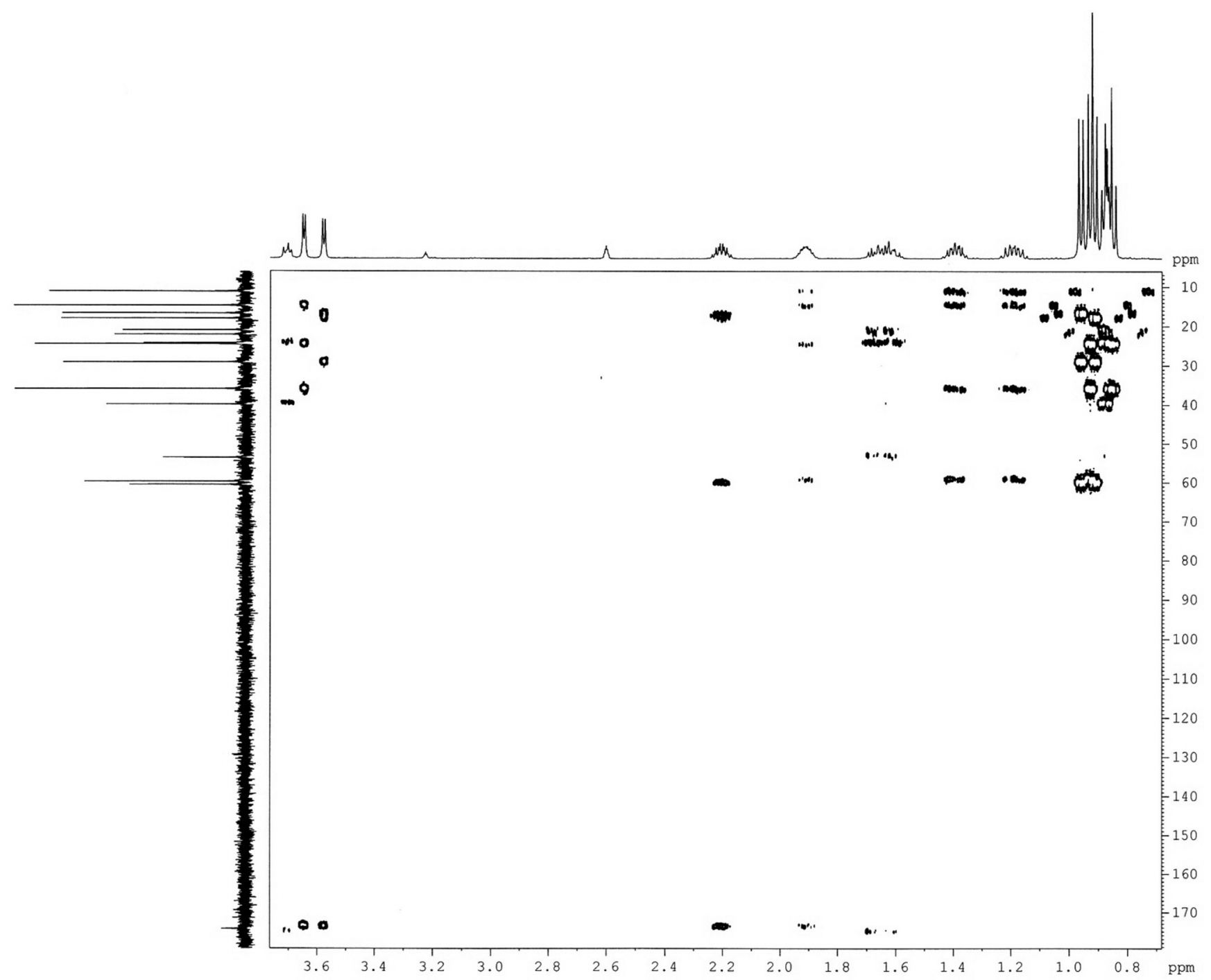

Figura 5S. Mapa de contornos $\mathrm{HMBC}\left(\mathrm{D}_{2} \mathrm{O}\right)$ do composto 6 


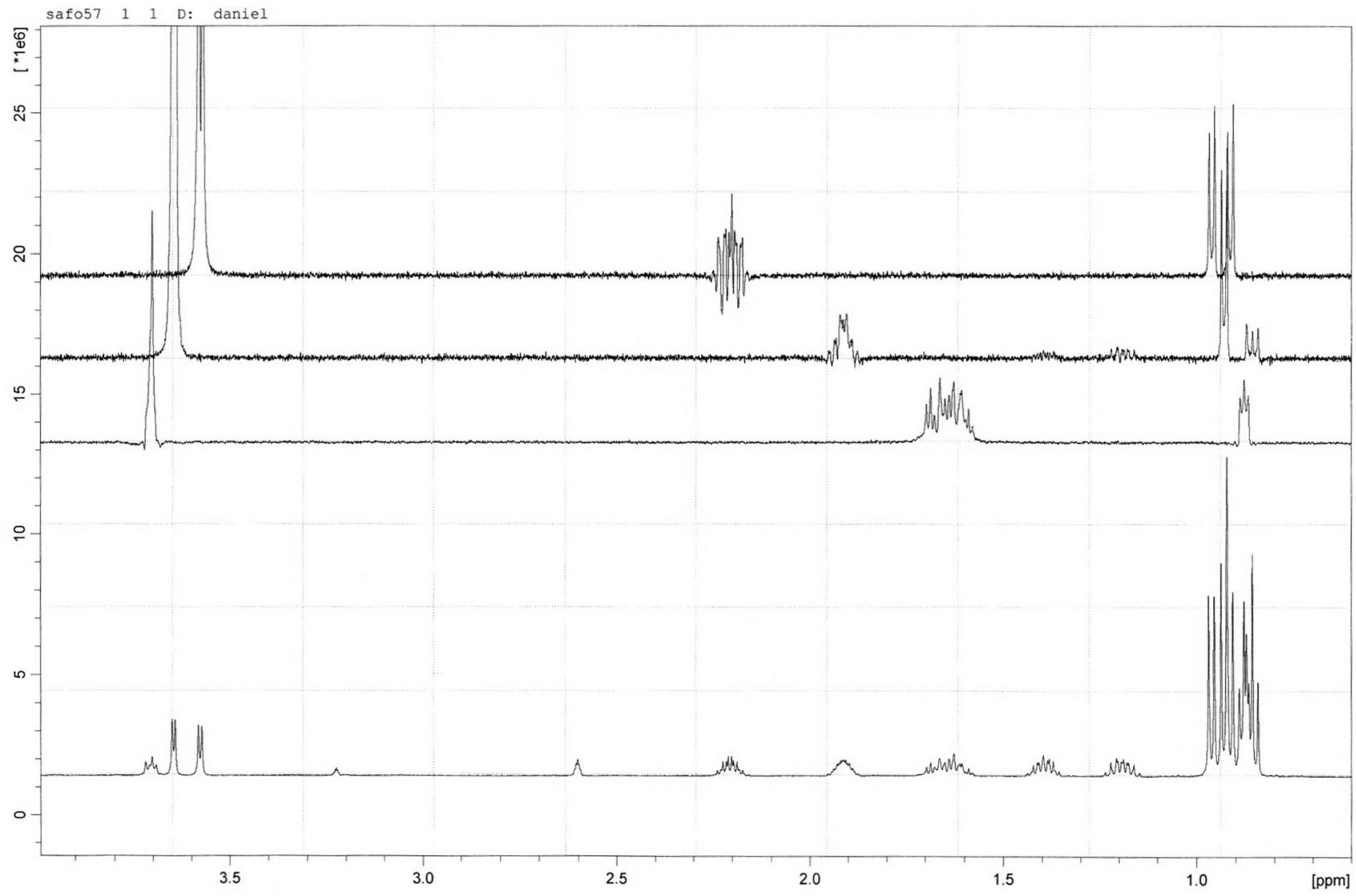

Figura 6S. Espectro de RMN TOCSY $1 D$ seletivo $\left(\mathrm{D}_{2} \mathrm{O}\right)$ do composto 6 